The reliability of supra-patellar transverse sonographic assessment of femoral trochlear cartilage thickness in healthy adults

Roberts, Harry; Moore, Jonathan; Thom, Jeanette

\title{
Journal of Ultrasound in Medicine
}

DOI:

10.1002/jum. 14775

Published: 01/04/2019

Peer reviewed version

Cyswllt i'r cyhoeddiad / Link to publication

Dyfyniad o'r fersiwn a gyhoeddwyd / Citation for published version (APA):

Roberts, H., Moore, J., \& Thom, J. (2019). The reliability of supra-patellar transverse sonographic assessment of femoral trochlear cartilage thickness in healthy adults. Journal of Ultrasound in Medicine, 38(4), 935-946. https://doi.org/10.1002/jum.14775

\footnotetext{
Hawliau Cyffredinol / General rights

Copyright and moral rights for the publications made accessible in the public portal are retained by the authors and/or other copyright owners and it is a condition of accessing publications that users recognise and abide by the legal requirements associated with these rights.

- Users may download and print one copy of any publication from the public portal for the purpose of private study or research.

- You may not further distribute the material or use it for any profit-making activity or commercial gain

- You may freely distribute the URL identifying the publication in the public portal ?
}

Take down policy

If you believe that this document breaches copyright please contact us providing details, and we will remove access to the work immediately and investigate your claim. 
1 The reliability of supra-patellar transverse sonographic assessment of femoral trochlear cartilage thickness in healthy adults

3

4 Running title: Femoral cartilage thickness evaluated by sonography

5

6 Harry M. Roberts Ph.D. ${ }^{1,2}$, Jonathan P. Moore Ph.D. ${ }^{1}$ \& Jeanette M. Thom Ph.D. ${ }^{1,3}$

7

$8 \quad{ }^{1}$ School of Sport, Health \& Exercise Sciences, Bangor University, UK

$9 \quad{ }^{2}$ School of Biosciences and Medicine, University of Surrey, UK

$10 \quad{ }^{3}$ School of Medical Sciences, University of New South Wales, Australia

12 Corresponding author:

13 Harry Roberts Ph.D.

14 University of Surrey

15 School of Biosciences and Medicine

16 The Leggett Building

17 Daphne Jackson Road

18 Guildford

19 GU2 7WG

20 Phone: $+44(0) 1483688687$

$21 \quad$ Email: h.m.roberts@surrey.ac.uk 


\section{Abstract}

\section{Objectives}

30 To determine the intra-session reliability of femoral cartilage thickness measurements using ultrasonography

31 and extend the pool of normative data for cartilage thickness measurements assessed by ultrasonography.

\section{Methods}

3377 healthy participants ( 55 male and 22 female), with an average age of $43 \pm 18$ (mean $\pm \mathrm{SD}$ ) years, volunteered.

34 Resting supra-patellar ultrasound was used to image trochlear cartilage thickness on two separate occasions a 35 maximum of 7 days apart. Reliability was evaluated with intraclass correlation coefficients (ICC), Bland \& 36 Altman analysis, standard error of measurement (SEM and SEM\%), and the smallest real difference (SRD and 37 SRD\%). Normative data was assessed using linear, multiple regression models and independent group t-tests.

\section{Results}

39 The test-retest level of agreement at all locations was high (ICC 0.779-0.843), which increased to high-very 40 high in young adults (ICC 0.884-0.920). The SEM\% was 8.2-8.3\% at all locations and reduced further to 5.4$416.3 \%$ in younger adults. The SRD\% was between $22.8-23.1 \%$ for the full sample and $14.9-17.5 \%$ in young 42 adults only. Multiple regression analyses demonstrated that age, weight, female gender and a high physical 43 activity frequency could significantly predict cartilage thickness at all locations $(\mathrm{P}<0.05)$; however, female 44 gender was the only significant independent predictor in all models (all $\mathrm{P}<0.01$ ). Females also had thinner 45 cartilage at all locations $(\mathrm{P}<0.01)$.

\section{Conclusion}

47 Supra-patellar ultrasonography demonstrates high intra-tester reliability and measurement precision and is a 48 promising method to assess trochlear cartilage thickness. Being female may impact femoral cartilage thickness 49 more than other potential risk factors for knee osteoarthritis such as age, weight, and high physical activity 50 frequency.

52 Keywords: Ultrasonography, femoral trochlear cartilage thickness, reproducibility of results, normative data 
Introduction

58 In recent years, ultrasound (US) has been increasingly used to assess cartilage thickness. A commonly adopted 59 technique is axial supra-patellar US imaging (1-6), although longitudinal US scanning of the knee has also been 60 used $(7,8)$. Despite its emergence as a method to assess trochlear cartilage thickness, to date, only a few studies 61 report the validity (through the comparison of US measurements with cadaver specimens or MRI imaging) and 62 reliability of sonographic evaluation of cartilage (7-9). Previous studies using US to measure trochlear cartilage 63 thickness have utilized either a young adult sample $(4,5)$, or have been confined to clinical populations $(2,3,10)$. 64 Therefore, the value of sonographic measurement of trochlear cartilage thickness in a healthy adult sample is restricted by limited normative data. Further examination of the accuracy and repeatability of this technique is required to establish whether US can be used as an effective tool to measure trochlear cartilage thickness. Overall, the ability to reliably measure trochlear cartilage thickness may offer an important tool to for the assessment of patellofemoral disorders, such as patellofemoral pain syndrome, chondromalacia patella and patellofemoral knee arthritis, in both a clinical and research setting.

The primary aim of this study was to assess the intra-session reliability of cartilage thickness measurements using sonography. Measurement precision was also assessed to identify the smallest change that can be considered actual change and not just a result of a test-re-test error. A secondary aim of this study was to also extend the pool of normative healthy adult data for cartilage thickness measurements assessed by ultrasonography.

\section{Materials and methods}

Seventy-seven healthy volunteers (55 male and 22 female), with an average age of $43 \pm 18$ years, and with an average body mass index (BMI) of $24.9 \pm 3.2$, were enrolled. Participants were targeted through word of mouth, poster advertisement, generic emails, and social media from the Bangor University community and the surrounding North Wales area. The inclusion criteria of entry to the study included being: (i) healthy, (ii) male or female, (iii) aged between 18-80 years. Exclusion criteria included: (i) diagnosed osteoarthritis (OA), rheumatoid arthritis, or other inflammatory diseases, (ii) history of knee malalignment (varus / valgus) greater than $15^{\circ}$, (iii) previous knee injury (including meniscus tear or ligament damage or tear), (iv) recent fracture of 
87 injections, or high dose oral steroids (vi) current or past (within last four weeks) glucosamine and/or chondroitin 88 supplementation use, (vii) pregnancy. This study was approved by the local Research Ethics Committee (School 89 of Sport Health and Exercise Sciences (SSHES), Bangor University) and conducted in accordance with the 90 Helsinki Declaration (2013). Written informed consent was obtained from all participants.

92 Experimental protocol

93 Participants were required to visit the School of Sport, Health and Exercise Science, Bangor University on two 94 occasions with each session lasting approximately 60 minutes. During the initial visit, participants completed a 95 medical and basic physical activity questionnaire. Anthropometric measurements (body mass and height) were 96 also assessed using a calibrated balance beam scale (SECA, California, USA) and wall-mounted tape measure 97 (SECA, California, USA), respectively. Ultrasonography was subsequently used to obtain images of the femoral 98 articular cartilage as outlined below. All participants completed their first and second visit at the same time of day and within a 7-day period. Participants were also asked to refrain from any strenuous physical activity for 48 hours prior to each visit.

101

102 Ultrasonography

103 The ultrasound (US) assessment was performed using a $12 \mathrm{MHz}$ linear-array probe (Esaote S.P.A. MyLab50 104 ultrasound, Firenze, Italy) and acoustic coupling gel (Aquasonic 100, Parker Laboratories, Inc, Fairfield, NJ, 105 USA) following a period of between 15-30 minutes of seated rest. With participants lying in a supine position, and with the knee maximally flexed, the superior margin of the patellar was located and a line was marked on the skin using a washable marker at the point immediately above the superior margin of the patellar and at $1 \mathrm{~cm}$ intervals in a superior direction. The transducer was placed in a supra-patella transverse position, perpendicular

109 to the bone surface and orientated to optimize the US image $(5,9)$. The location at which the cartilage thickness

110 of the intercondylar notch appeared greatest was marked on the skin and recorded to enable the examiner to 111 return the transducer to the exact location for all subsequent scans. The same researcher performed all 112 ultrasonography scans following training by a consultant rheumatologist with expertise using this technique.

114 US images were analyzed by 'Image J' software (Image J, National Institute of Health, Bethesda, MD, USA) to 115 determine the minimal cartilage thickness. The distance from the thin hyperechoic line formed at the synovial 116 space-cartilage border to the line formed at the cartilage-bone border was used to measure minimal cartilage 
117 thickness at the lateral facet, medial facet and intercondylar notch. Anatomic reference points used in the present 118 study corresponded to the midpoint of the intercondylar notch and $1 \mathrm{~cm}$ apart in the medial and lateral directions 119 were used as an estimate of the cartilage thickness at the medial and lateral facet, respectively (11). Naredo and 120 colleagues previously demonstrated good reproducibility in femoral cartilage thickness measurement when 121 using comparable anatomical reference points (5). Prior to analysis, all images were de-identified by a second 122 researcher for blinded analysis. Based on the pixel resolution $(15.8$ pixels $/ \mathrm{mm})$ of the images captured by 123 ultrasonography, the ImageJ software allowed images to be measured to an accuracy of greater than one-tenth 124 off a $\mathrm{mm}$, or more specifically, one pixel was equal to $0.06 \mathrm{~mm}$. The cartilage thickness of each image was 125 measured in triplicate and an average of the three measurements was used for all data analysis. As required, the 126 image contrast was adjusted to assist in appropriately identifying the hyperechoic line formed at the synovial space-cartilage border to the line formed at the cartilage-bone border.

129 Statistical analysis

130 Reliability analysis

131 Agreement between measurements was evaluated using a one-way mixed, absolute agreement type, intraclass 132 correlation coefficient (ICC) (12). ICC values can be classified as low: $0.20-0.49$; moderate: $0.50-0.69$; high: $1330.70-0.89$; or very high: $0.90-1.00$ (13). Paired t-tests, together with Bland-Altman plots, were used to provide 134 an indication of the systematic error (14). The standard error of the measurement (SEM) and the SEM\% were 135 calculated as previously described $(15,16)$. SEM and the SEM\% were used to establish the measurement 136 precision between visit 1 and 2 and to provide a measure of the smallest value that represents a real change in a 137 group of individuals. Furthermore, to calculate the smallest error in a single individual score, the smallest real 138 difference (SRD) and SRD\% were also calculated as previously described (17). All analyses were initially 139 completed using the full dataset. Finally, a split-group analysis was performed for each of the following groups: 140 young adults (18-25 years of age), middle-aged adults (26-50 years of age), older adults ( $\geq 51$ years of age), 141 male only, and female only groups. This analysis provided an opportunity to determine whether age or sex of the participant influenced the level of intra-tester reliability and measurement precision.

145 Independent t-tests were used to determine differences between measurements made on the right and 146 measurements made on the left knee. If data was not available for either the right or left side (i.e. a measurement 
147 could not be made for one of the locations), both visit 1 and visit 2 data points were removed to ensure an equal 148 sample size. Simple linear regression analyses were performed to determine the relationship between mean 149 cartilage thickness of the right knee (at each location) and participant characteristics (age, body mass, height, and BMI). Multiple linear regression models were subsequently used to explore the relationship between

151 cartilage thickness (at each location) and potential risk factors; including age, BMI, and female gender and high 152 frequency of weekly physical activity. Physical activity was considered 'high' when participants completed 153 structured exercise training on a minimum of 5 days per week. In addition to multiple regression, mean cartilage 154 thickness between sexes was also assessed by creating an equal sized $(n=17)$ sample matched for age and BMI. 155 Independent t-tests were used to determine whether cartilage thickness differences existed between males and 156 females at each measurement location (intercondylar notch, lateral facet, medial facet). For the multiple comparisons of cartilage thickness between the three locations, Bonferroni corrections were used with $\mathrm{P}<0.016$

$158(0.05 / 3)$ for statistical significance. Finally, a one-way analysis of variance (ANOVA) was used to assess the differences in cartilage thickness measurements at each measurement location. The left side was not used within

160 the analysis of normative data as the side to side differences were found to be small and within measurement error in the present study. Moreover, others have reported limited side-to-side differences in femoral cartilage thickness measurement (18) and have advocated the use of unilateral OA models in research (19).

\section{Results}

165 The results from the US measurement of cartilage thickness and participant characteristics are displayed in

166 Table 1. A total of 308 knees were scanned (right and left knee of 77 participants on two occasions). This 167 produced a total of 1168 blinded images (77 participants were imaged three to four times per side on two 168 occasions, i.e. visit 1 and visit 2). Some individual images could not be measured as the hyperechoic line 169 formed at the synovial-cartilage border and/or cartilage-bone border could not be clearly delineated. Thus, it was not possible to confidently measure cartilage thickness for $129(11 \%), 180(15 \%)$, and $221(19 \%)$ of the available images for the intercondylar notch, medial and lateral facet, respectively. For the cartilage thickness reliability to be assessed a minimum of one image per location was required. Overall, cartilage thickness could

173 be measured in 306 knees $(99.4 \%)$ at the medial facet, 304 knees $(98.7 \%)$ at the intercondylar notch, and 296 knees $(96.1 \%)$ at the lateral facet.

\section{Reliability analysis}


177 The ICC and 95\% confidence intervals (95\% CI) for the data are shown in Table 2. The ICC's indicate that the 178 level of agreement at all locations was high (ICC between $0.779-0.843$ ), with the highest at the intercondylar 179 notch, followed by the medial facet and then the lateral facet. Subsequent analyses revealed that the level of agreement between measurements was considerably improved when considering younger participants $(\leq 25$ years of age) only (Table 2). In addition, the image quality and clarity were typically better in younger individuals as highlighted in Figure 1. Results also demonstrated that the intra-tester reliability of cartilage thickness measurements was generally similar when male and female groups were analyzed separately (Table 2).

185 Systemic variation in cartilage thickness measurements at the notch, medial facet, and lateral facet are shown by 186 Bland-Altman plots (Figure 2). The plots suggest that slightly higher variation (i.e. heteroscedasticity) may exist 187 for higher cartilage thickness measurements, particularly at the notch and medial facet. Moreover, results of the 188 paired t-tests showed no significant difference in cartilage thickness between visit 1 and visit 2 for the medial $189(1.83$ vs $1.82 \mathrm{~mm} ; \mathrm{P}=0.760)$ and lateral facet locations $(1.81$ vs $1.81 \mathrm{~mm} ; \mathrm{P}=0.860)$. However, at the 190 intercondylar notch, a small but significantly greater cartilage thickness measurement was obtained at visit 2 , 191 thus indicating that measurements made during the second visit may be systematically higher compared to 192 measurements made at visit $1(2.03$ vs $2.08 \mathrm{~mm} ; \mathrm{P}=0.016)$. When data was split based on the age of the individuals, paired t-tests (visit 1 versus visit 2) did not reveal any systematic differences in measurements made

194 in young participants. Furthermore, although mean differences in cartilage thickness measurements tended to be slightly higher in middle-aged and older participants at most measurement locations compared to the young

196 group, a significant difference between visit 1 and visit 2 was only present at the intercondylar notch in the middle-aged participants.

199 The SEM is provided for each location in Table 2. The value ranged from $0.15-0.17 \mathrm{~mm}$ for all locations. In 200 agreement with the intra-class correlation analysis, the SEM was lowest in the split group analysis of young 201 participants only (Table 2). Moreover, the SEM\%, which provides a measure independent of units, indicates that 202 differences in groups of individuals above $8.2-8.3 \%$ can be considered a real change and not the difference 203 associated with measurement error. Overall, the SEM\% values for all analyses (Table 2) provide evidence of a 204 relatively low range $(5.4-9.6 \%)$. Moreover, the smallest real change is the measurement error in a single 205 individual cartilage thickness. Table 2 demonstrate that the SRD is between 0.42 and $0.47 \mathrm{~mm}$ for all locations. 
In relative terms, this equals $22.8-23.1 \%$. An improvement in the smallest real change was shown in young

participants $(0.28-0.32 \mathrm{~mm})$ and when analyzing females only $(0.27-0.34 \mathrm{~mm})$.

Sonographic assessment of cartilage thickness

210 Femoral cartilage thickness did not differ between the right and left intercondylar notch, or between the right

211 and left medial facet. Although differences were observed at the lateral facet between the left and right knee

212 (1.78 vs $1.88 \mathrm{~mm}, \mathrm{P}=0.04)$, the difference was small $(5.6 \%)$ and within the SEM. For this reason (and as

213 previously stated in the methodology), normative data analyses were based on the right side only.

215 Age was found to have a negative relationship with lateral cartilage thickness in men (Figure 3A). Participant 216 weight was found to have a positive relationship with cartilage thickness at the intercondylar notch (Figure 3C).

217 When females and men were assessed separately, this positive correlation was only found in men (Figure 4C).

218 Participant height was also found to have a positive relationship with cartilage thickness at all locations (Figure

219 3B); however, when the analysis was separated for males and females, a positive relationship remained between

220 height and lateral facet cartilage thickness in males only. Moreover, a negative relationship between height and

221 intercondylar notch thickness was found in females. In addition, BMI was found to have a negative relationship

222 with lateral and medial facet thickness in men, but not women (Figure 4D). The correlation coefficient, levels of

223 significance and regression equation are presented in Figure 3 for the full dataset, and in Figure 4 for the 224 comparison between males and females.

Age, weight, female gender and high physical activity frequency ( $>5$ sessions per week) were the independent variables included in the multiple regression model. This four-predictor model was able to account for $28.8 \%$ of the variance in femoral cartilage thickness at the intercondylar notch $\left[\mathrm{F}(4,59)=5.953, \mathrm{P}<0.001, R^{2}=0.288\right]$. However, gender was the only independent variable to significantly contribute to the model. The beta coefficient $(\beta=-0.367)$ indicates that in this sample the cartilage thickness in females was $0.38 \mathrm{~mm}$ lower than males $(\mathrm{P}<0.01)$. At the lateral facet, the regression model could predict $16 \%$ of the variance in cartilage thickness $\left[\mathrm{F}(4,60)=2.857, \mathrm{P}<0.05, R^{2}=0.160\right]$. As per the previous regression model, gender was the only independent variable to significantly contribute to the model $(\beta=-0.253, P=0.008)$. Finally, age, weight,

234 female gender and a high physical activity frequency at the medial facet could significantly predict $15.1 \%$ of the 
variance in cartilage thickness at the medial facet $\left.\mathrm{F}(4,60)=2.659, \mathrm{P}<0.05, R^{2}=0.151\right]$. However, again,

gender was the only independent variable that contributed to the model $(\beta=-0.355, \mathrm{P}=0.03)$.

238 Analysis of the stable model, i.e. with sex (a dummy variable) as the only predictor variable, the following 239 models were produced: For the femoral intercondylar notch, femoral cartilage thickness in females could be 240 calculated as $2.204+(-0.450 \times 1)=1.754 \mathrm{~mm}$, and in males it could be calculated as $2.204+(-0.450 \times 0)=$ $2412.204 \mathrm{~mm}$. The model was significant $(\mathrm{P}<0.001)$. For the femoral lateral facet, femoral cartilage thickness in 242 females could be calculated as $1.933+(-0.219 \times 1)=1.714 \mathrm{~mm}$, and in males it could be calculated as $1.933+$ $243(-0.219 \times 0)=1.933 \mathrm{~mm}$. This model was also significant $(\mathrm{P}<0.01)$. Finally, for the femoral medial facet, 244 femoral cartilage thickness in females could be calculated as $1.946+(-0.295 \times 1)=1.651 \mathrm{~mm}$, and in males it could be calculated as $1.946+(-0.295 \times 0)=1.946 \mathrm{~mm}$. This model was also significant $(\mathrm{P}<0.01)$.

Results demonstrated that cartilage thickness was thicker at the intercondylar notch compared to the medial and lateral facet (Figure 5). However, there was no difference in cartilage thickness between the medial and lateral facet $(\mathrm{P}>0.05)$. To further assess for differences in mean cartilage thickness between sexes an equal sized $(\mathrm{n}=$ 17) sample matched for age and BMI was created. Results demonstrated that mean cartilage thickness was lower in females at the intercondylar notch, lateral facet and medial facet than that of the matched male group (Figure 6). The biggest difference in mean cartilage thickness between males and females was at the medial facet (2.00 $\mathrm{mm}$ versus $1.60 \mathrm{~mm}$, respectively).

\section{Discussion}

256 The use of supra-patellar transverse sonography to assess trochlear cartilage thickness is a novel technique, which required the further study into its reliability and accuracy. The purpose of the present study was to ascertain the intra-tester reliability of supra-patellar transverse US of trochlear cartilage thickness in a group of healthy males and females across a wide range of ages. Notably, the present study demonstrates high intra-tester reliability for trochlear cartilage thickness at the intercondylar notch, medial facet, and lateral facet, as well as a reasonably small measurement error. Additional analysis revealed that both intra-tester reliability and counterparts. 
265 In healthy individuals, supra-patellar transverse ultrasonography allowed a quick and straightforward assessment of trochlear cartilage. The high ICCs found in the present study [intercondylar notch $0.843(0.790$ $0.883)$, medial facet $0.834(0.778-0.835)$ and lateral facet $0.779(0.707-0.876)]$, are comparable to previously reported ICCs using a very similar standardized protocol in a small sample of flexed cadaver knee (age of death was 76-89 years) (9). Interestingly, in both studies, the level of agreement at the lateral facet was lower compared to the intercondylar notch. One possibility is that the lateral and medial facets are prone to an increased level error related to the inclination and positioning of the US transducer (9). This is supported by previous evidence using MRI, which reported that central weight regions often provide greater accuracy than

273 boundary areas (20). Results in the present study also revealed that intra-tester reliability was considerably 274 greater in younger individuals compared to middle-aged and older individuals. Given that a limited degenerative change would be expected in young healthy adults, the increased reliability in young individuals might be due to the trochlear cartilage appearing considerably clearer in young participants. In contrast, image quality in older individuals was often lower, thus reducing the ability of the investigator to delineate images and offer such precise measurements.

280 Images obtained from this study provided a clear hyperechoic line formed at the synovial-cartilage border 281 and/or cartilage-bone border that allowed femoral cartilage thickness to be assessed in most but not all cases.

282 Compared to the study by Yoon and colleagues, cartilage thickness could be measured in a greater proportion of 283 knees at the medial facet (98.7 vs $70.6 \%$ ) and lateral facet (96.1 vs 90.1\%) in the current study (8). Differences 284 in the ability to measure cartilage thickness between the two studies are likely to relate to the participants (i.e. 285 OA vs healthy individuals in the current study). Several degenerative changes, including, roughened and 286 fibrillated articular cartilage, cartilage loss, asymmetrical narrow, as well as abnormalities at the subchondral 287 bone have previously been associated with poorly defined hyperechoic cartilage borders $(5,8)$. Moreover, 288 despite great care being used to standardize the US assessment of the knee and to replicate the positioning of 289 both the participant and transducer between sessions, other factors such as poor transducer positioning or movement artefact, may also contribute to poor image quality (9). In the current study, of the 7 knees which could not be measured, individuals were all male, mostly older and had a higher BMI. These factors and the relationship with the ability to measure cartilage thickness using US warrant further investigation. 
294 Femoral trochlear cartilage thickness measurements in the present study were comparable to several previous 295 studies using the same US methodology in similarly aged healthy individuals $(3,21)$. In contrast, others have 296 reported slightly greater femoral trochlear cartilage thicknesses in young (25-40 years) healthy individuals 297 compared to the present study (5). The present study also found a significantly thicker cartilage thickness at the 298 intercondylar notch compared to the lateral and medial facets. This difference has not been observed to the same 299 extent in several other studies $(3,5,21)$ and may be related to differences in biomechanical loading. In addition, 300 femoral trochlear cartilage thickness did not differ between the right and left intercondylar notch, or the right 301 and left medial facet. Although differences were observed at the lateral facet between the left and right knee 302 (1.78 vs $1.88 \mathrm{~mm}, \mathrm{P}=0.04)$, the difference was small $(5.6 \%)$ and within the SEM. Side to side differences in 303 thickness have previously been reported; however, differences in cartilage thickness tend to be small (total knee 304 joint: $3.8 \pm 3.1 \%$ ) with no significant differences for limb dominance (22). A previous report indicates good 305 correlations between morphological dimensions of the left and right side and advocates the use of unilateral OA models in research (19).

The current study also found females had lower cartilage thickness at all locations compared to males, which is 309 consistent with previous studies using both MRI and US $(5,23,24)$. Furthermore, regression analyses in the 310 present study found that female gender was the only variable that could explain the variation in cartilage 311 thickness. The lower trochlear cartilage thicknesses observed in the present study may relate to differences in 312 body size between men and women. This is supported by the current finding that women have thinner trochlear 313 cartilage thickness compared to men after the adjustment for age and BMI (Figure 6), and previously, after 314 adjustment for body height and weight (24). Differences between males and females may also relate to 315 differences in the sex hormone estrogen (25), which is understood to act upon estrogen receptors found in 316 articular cartilage (26), and/or to differences in the dynamic loading across the knee joint between men and 317 women (27).

319 Further analyses demonstrated that age was negatively associated with cartilage thickness at the lateral facet, but 320 only in males. Similarly, several studies have previously found ageing to be negatively associated with femoral 321 cartilage thickness assessed by both US (5) and MRI (28). The results of the current study suggest that the 322 lateral femoral facet might be the most prominent site for age-related change. Furthermore, although age has 323 previously been found to be negatively associated with femoral cartilage thickness in both men and women (5), 
324 the present study suggests that men are more at risk of age-related change in cartilage thickness at the lateral

325 facet. This finding is surprising given that older women are at increased risk of OA (29) and may relate to the 326 small sample of females in the present study.

328 Anthropometric variables such as body height and weight may also influence trochlear cartilage thickness. In 329 the current study, a positive relationship was found between body height and cartilage thickness for all three 330 locations. Several previous studies have also found body height to be positively associated, albeit weakly, with 331 cartilage thickness $(24,28)$. In contrast, a positive relationship between weight and cartilage thickness was 332 observed only at the intercondylar notch. Moreover, when the relationship was explored separately for males 333 and females, weight and body height demonstrated a different relationship with femoral cartilage thickness.

334 Both body height and weight were shown to have a positive relationship with cartilage thickness at various 335 locations in men, while in females, weight was unrelated to femoral cartilage thickness and body height was 336 negatively related at certain locations. Previous research also demonstrated that neither weight nor height was 337 correlated with femoral cartilage thickness in women, and only body height was positively correlated with 338 femoral cartilage thickness in men (30). Reasons for the difference between men and women are unknown. 339 However, it appears that the higher joint loads that are related to body size may have a more favorable impact 340 on cartilage thickness of healthy men compared to women. Whether the relationship extends to a group of men 341 with a greater variation in body size remains unclear. The present study also found that BMI had a negative 342 relationship with both lateral and medial facet thickness in men. This supports previous research indicating 343 having a high BMI may increase the risk of reduced cartilage thickness and knee OA (31). The results of the 344 current study would suggest that while being either heavier or taller may be positive for cartilage thickness in 345 men, an unfavorable body composition may reduce cartilage thickness. This may also suggest that muscle 346 function and physical fitness may have a key role in cartilage thickness morphology. Although exercise 347 frequency as a measure of physical activity level was not associated with cartilage thickness in the present 348 study, future research, together with more refined measures of physical activity is required to explore the potential relationship and determine whether a moderation effect exists.

351 A primary limitation of this study was the inability to determine the validity of femoral cartilage thickness 352 measurements made using US with a gold standard such as MRI. Nonetheless, US may be regarded as a 353 promising measurement technique that has demonstrated a good agreement in both cartilage thickness 
measurements made using US and MRI (7) as well as US and anatomical specimens (9). Nevertheless, this level

of agreement is not a universal finding, particularly when using supra-patellar axial US to assess medial facet thickness (8) and when severely damaged knees are included in the analysis (9). Importantly, caution is warranted when considering the validity and reliability of sonographic measures of cartilage thickness when the sample includes older individuals, and individuals with significant knee OA. In addition, unlike the analysis of MRI, US cartilage thickness measurements are largely limited to the femoral plate and do not offer the ability to assess other morphological measurements such as cartilage volume. Unlike MRI, the use of US to detect changes in cartilage thickness following acute loading is unclear. Although acute changes in femoral cartilage thickness following walking and running have recently been reported (32), our recent work demonstrated that such change was not a universal finding (11). A further limitation of the present study relates to the fact that inter-tester reliability was not assessed. This is particularly important given the usefulness of sonographic cartilage thickness measurements as a clinical and research tool relies on the ability to make direct comparisons between studies.

This cross-sectional study of the healthy adults, with a wide age range, demonstrates high intra-tester reliability

369 for all femoral cartilage locations (ICC's between 0.779-0.843) and measurement precision (SEM\% between

370 8.2-8.3\%), which is better in younger adults (ICC's between 0.884-0.920 and SEM\% 5.4-6.3\%). Thus, in 371 younger adults, differences between groups or because of an intervention, that is greater than $6.3 \%$ would 372 represent real difference and not just measurement error. Finally, this study also provides normative data for 373 knee cartilage thickness measured by sonography. Considerable variability exists in the femoral cartilage 374 thicknesses of healthy individuals. However, cartilage thickness appears greatest at the intercondylar notch 375 compared to the medial and lateral facets. Furthermore, the data suggest that females have reduced cartilage 376 thickness compared with males and that both ageing and anthropometric measures affect cartilage thickness differently in males and females. This research offers interesting data for the study of the patello-femoral joint.

\section{Acknowledgements}

380 The authors thank all the participants who volunteered for this study.

\section{References}

383 1. Akkaya S, Akkaya N, Ozcakar L, Kilic A, Sahin F, Atalay NS, et al. Ultrasonographic evaluation of the 
femoral cartilage thickness after unilateral arthroscopic partial meniscectomy. Knee Surg Sports Traumatol Arthrosc. 2013 May; 21(5):1104-1110.

386 2. Kara M, Tiftik TTT, Öken Ö, Akkaya N, Tunc H, Özçakar L, et al. Ultrasonographic measurement of femoral cartilage thickness in patients with spinal cord injury. J Rehabil Med. 2013; 45(2):145-148.

388 3. Kaya A, Kara M, Tiftik TTT, Tezcan ME, Ozturk MA, Akinci A, et al. Ultrasonographic evaluation of the femoral cartilage thickness in patients with systemic lupus erythematosus. Rheumatol Int. $2012 \mathrm{Apr}$; 33(4):899-901. Malas FÜU, Kara M, Aktekin L, Ersoz M, Ozcakar L, Ersöz M, et al. Does vitamin D affect femoral cartilage thickness? An ultrasonographic study. Clin Rheumatol. 2013 Sep; 33(9):1-4.

5. Özçakar L, Tunç H, Öken Ö, Ünlü Z, Durmu B, Ozcakar L, et al. Femoral cartilage thickness measurements in healthy individuals: learning, practicing and publishing with TURK-MUSCULUS. $J$ Back Musculoskelet Rehabil. 2014;27(2):117-124.

6. Ozturk GT, Malas FÜU, Yildizgoren MT, Baki AE, Inal EE, Batmaz İIIIII, et al. Ultrasonographic Assessment of the Femoral Cartilage Thickness in Patients with Pes Planus. Am J Phys Med Rehabil. $2015 \mathrm{Jul} ; 94(7): 568-572$.

7. Tarhan S, Unlu Z, Goktan C. Magnetic resonance imaging and ultrasonographic evaluation of the patients with knee osteoarthritis: a comparative study. Clin Rheumatol. 2003; 22(3):181-188. sonographic longitudinal sagittal image for assessment of the cartilage thickness in the knee osteoarthritis. Clin Rheumatol. 2008;27(12):1507-1516.

9. Naredo E, Acebes C, Möller I, Canillas F, de Agustín JJ, de Miguel E, et al. Ultrasound validity in the measurement of knee cartilage thickness. Ann Rheum Dis. 2009;68(8):1322-1327. measurement of the distal femoral cartilage thickness in patients with unilateral transtibial amputation. Prosthet Orthot Int. 2013; 37(4):268-274. 
414 13. Munro B. Correlation. In: Munro B, editor. Statistical methods for health care research. 5th ed. Philadelphia: Lippincott, Williams, and Wilkins; 2005: p. 239-258.

416 14. Atkinson G, Nevill A. Statistical methods for assessing measurement error (reliability) in variables relevant to sports medicine. Sport Med. 1998;26(4):217-238.

15. Thomas JR, Nelson KJ. Research methods in physical activity. Champaign (IL): Human Kinetics; 1990.

16. Beckerman H, Roebroeck ME, Lankhorst GJ, Becher JG, Bezemer PD, Verbeek ALM. Smallest real difference, a link between reproducibility and responsiveness. Qual Life Res. 2001;10(7):571-578.

17. Eliasziw M, Young SL, Woodbury MG, Fryday-Field K. Statistical methodology for the concurrent assessment of interrater and intrarater reliability: using goniometric measurements as an example. Phys Ther. 1994;74(8):777-788.

18. Eckstein F, Müller S, Faber SC, Englmeier KH, Reiser M, Putz R. Side differences of knee joint cartilage volume, thickness, and surface area, and correlation with lower limb dominance - An MRIbased study. Osteoarthr Cartil. 2002;10(12):914-921.

19. Dargel J, Feiser J, Gotter M, Pennig D, Koebke J. Side differences in the anatomy of human knee joints. Knee Surgery, Sport Traumatol Arthrosc. 2009;17(11):1368-1376.

20. Koo S, Gold GEE, Andriacchi TPP. Considerations in measuring cartilage thickness using MRI: factors influencing reproducibility and accuracy. Osteoarthr Cartil. 2005;13(9):782-789.

21. Malas FU, Kara M, Kaymak B, Akinci A, Ozcakar L. Ultrasonographic evaluation in symptomatic knee osteoarthritis: clinical and radiological correlation. Int J Rheum Dis. 2014;17(5):536-540.

433 22. Eckstein F, Faber S, Mühlbauer R, Hohe J, Englmeier K-H, Reiser M, et al. Functional adaptation of human joints to mechanical stimuli. Osteoarthr Cartil. 2002;10(1):44-50.

435 23. Faber SC, Eckstein F, Lukasz S, Mühlbauer R, Hohe J, Englmeier KH, et al. Gender differences in knee joint cartilage thickness, volume and articular surface areas: assessment with quantitative threedimensional MR imaging. Skeletal Radiol. 2001;30(3):144-150.

438 24. Otterness IG, Eckstein F. Women have thinner cartilage and smaller joint surfaces than men after adjustment for body height and weight. Osteoarthr Cartil. 2007;15(6):666-672.

440 25. Ben-Hur H, Thole HH, Mashiah A, Insler V, Berman V, Shezen E, et al. Estrogen, progesterone and testosterone receptors in human fetal cartilaginous tissue: immunohistochemical studies. Calcif Tissue Int. 1997;60:520-560.

26. Ushiyama T, Ueyama H, Inoue K, Ohkubo I, Hukuda S. Expression of genes for estrogen receptors 
alpha and beta in human articular chondrocytes. Osteoarthr Cartil. 1999;7:560-566.

27. Cicuttini F, Forbes A, Morris K, Darling S, Bailey M, Stuckey S. Gender differences in knee cartilage volume as measured by magnetic resonance imaging. Osteoarthr Cartil. 1999;7(3):265-271.

28. Hudelmaier M, Glaser C, Hohe J, Englmeier KH, Reiser M, Putz R, et al. Age-related changes in the morphology and deformational behavior of knee joint cartilage. Arthritis Rheum. 2001;44(11):25562561.

450 29. Felson DT, Naimark a, Anderson J, Kazis L, Castelli W, Meenan RF. The prevalence of knee osteoarthritis in the elderly. The Framingham Osteoarthritis Study. Arthritis Rheum. 1987;30(8):914918.

30. Hudelmaier M, Glaser C, Englmeier K-H, Reiser M, Putz R, Eckstein F. Correlation of knee-joint cartilage morphology with muscle cross-sectional areas vs. anthropometric variables. Anat Rec A Discov Mol Cell Evol Biol. 2003;270(2):175-184.

31. Reijman M, Pols HA, Bergink AP, Hazes JM, Belo JN, Lievense AM, et al. Body mass index associated with onset and progression of osteoarthritis of the knee but not of the hip: the Rotterdam Study. Ann Rheum Dis. 2007;66(2):158-162. Assessment of Medial Femoral Cartilage Deformation Acutely Following Walking and Running. 
Table Error! No text of specified style in document. Physical characteristics of participants and knee cartilage thickness

\begin{tabular}{|c|c|c|c|c|c|c|}
\hline & \multicolumn{2}{|c|}{ Men $(n=55)$} & \multicolumn{2}{|c|}{ Women $(n=22)$} & \multicolumn{2}{|c|}{ Total $(n=77)$} \\
\hline & Mean (SD) & Range & Mean (SD) & Range & Mean (SD) & Range \\
\hline Age (years) & $45(18)$ & $18-70$ & $38(20)$ & $20-79$ & $43(18)$ & 18- 79 \\
\hline Body height (m) & $1.77(0.06)$ & $1.64-1.95$ & $1.67(0.06)$ & $1.51-1.78$ & $1.74(0.08)$ & $1.5-1.95$ \\
\hline Body mass (kg) & $79.7(11.1)$ & $63.3-120.7$ & $66.3(11.6)$ & $40.5-89.4$ & $75.8(12.7)$ & $40.5-120.7$ \\
\hline BMI & $25.4(3.0)$ & $21.0-35.7$ & $23.7(3.5)$ & $17.7-30.2$ & $24.9(3.2)$ & $17.7-35.7$ \\
\hline \multicolumn{7}{|c|}{ Knee cartilage thickness (mm) } \\
\hline \multicolumn{7}{|l|}{ Right } \\
\hline Lateral & $1.93(0.29)$ & $1.43-2.73$ & $1.71(0.29)$ & $1.23-2.37$ & $1.87(0.30)$ & $1.23-2.73$ \\
\hline Notch & $2.20(0.40)$ & $1.28-3.22$ & $1.75(0.23)$ & $1.27-2.36$ & $2.07(0.43)$ & $1.27-3.22$ \\
\hline Medial & $1.95(0.38)$ & $1.15-2.97$ & $1.65(0.28)$ & $1.06-2.30$ & $1.86(0.38)$ & $1.06-2.97$ \\
\hline \multicolumn{7}{|l|}{ Left } \\
\hline Lateral & $1.79(0.30)$ & $1.02-2.37$ & $1.68(0.34)$ & $0.93-2.44$ & $1.76(0.32)$ & $0.93-2.44$ \\
\hline Notch & $2.17(0.40)$ & $1.44-3.12$ & $1.84(0.32)$ & $1.18-2.46$ & $2.08(0.41)$ & $1.18-3.12$ \\
\hline Medial & $1.84(0.34)$ & $1.01-2.60$ & $1.63(0.31)$ & $1.08-2.28$ & $1.78(0.34)$ & $1.01-2.60$ \\
\hline
\end{tabular}


478 Table 2 Reliability of cartilage thickness measurements made at visit 1 and visit 2 for all locations with

479 comparisons between age and gender

\begin{tabular}{|c|c|c|c|c|c|}
\hline Location & ICC (95\% CI) & SEM & SEM\% & SRD & SRD\% \\
\hline \multicolumn{6}{|c|}{ Overall $(n=77)$} \\
\hline Notch & $0.843(0.790-0.883)$ & 0.17 & 8.2 & 0.47 & 22.9 \\
\hline Medial & $0.834(0.778-0.876)$ & 0.15 & 8.2 & 0.42 & 22.8 \\
\hline Lateral & $0.779(0.707-0.835)$ & 0.15 & 8.3 & 0.42 & 23.1 \\
\hline \multicolumn{6}{|c|}{ Young adults $(n=20)$} \\
\hline Notch & $0.920(0.854-0.957)$ & 0.12 & 5.7 & 0.32 & 15.8 \\
\hline Medial & $0.884(0.792-0.937)$ & 0.11 & 6.3 & 0.32 & 17.5 \\
\hline Lateral & $0.906(0.830-0.949)$ & 0.10 & 5.4 & 0.28 & 14.9 \\
\hline \multicolumn{6}{|c|}{ Middle-aged adults $(\mathrm{n}=29)$} \\
\hline Notch & $0.843(0.747-0.905)$ & 0.18 & 8.4 & 0.49 & 23.3 \\
\hline Medial & $0.800(0.684-0.877)$ & 0.13 & 6.8 & 0.35 & 18.9 \\
\hline Lateral & $0.639(0.453-0.772)$ & 0.17 & 9.2 & 0.47 & 25.4 \\
\hline \multicolumn{6}{|c|}{ Older adults $(n=28)$} \\
\hline Notch & $0.779(0.651-0.864)$ & 0.19 & 9.2 & 0.51 & 25.4 \\
\hline Medial & $0.832(0.731-0.898)$ & 0.17 & 9.6 & 0.48 & 26.5 \\
\hline Lateral & $0.788(0.661-0.872)$ & 0.15 & 8.7 & 0.42 & 24.2 \\
\hline \multicolumn{6}{|c|}{ Male only $(n=55)$} \\
\hline Notch & $0.804(0.725-0.862)$ & 0.18 & 8.3 & 0.50 & 23.3 \\
\hline Medial & $0.803(0.725-0.861)$ & 0.16 & 8.7 & 0.45 & 24 \\
\hline Lateral & $0.744(0.645-0.819)$ & 0.15 & 8.2 & 0.42 & 22.8 \\
\hline \multicolumn{6}{|c|}{ Female only $(n=22)$} \\
\hline Notch & $0.838(0.723-0.908)$ & 0.12 & 6.9 & 0.34 & 19.0 \\
\hline Medial & $0.870(0.775-0.927)$ & 0.10 & 5.8 & 0.27 & 16.2 \\
\hline Lateral & $0.828(0.708-0.902)$ & 0.12 & 6.8 & 0.32 & 19.0 \\
\hline \multicolumn{6}{|c|}{$\begin{array}{l}\text { ICC }=\text { intra-class correlation; } C I=\text { confidence intervals; } S E M=\text { standard error of measurement; } S R D= \\
\text { smallest real difference); young }(\leq 25 \text { years of age }) \text {, middle-aged ( } 26-50 \text { years of age }) \text {, and old age group } \\
51 \text { years of age })\end{array}$} \\
\hline
\end{tabular}

480 
482 Figure 1 US transverse image of the femoral articular cartilage demonstrating the difference in image quality and clarity between young, middle-aged, and old groups. Image A) represents the 'young' group (23-year-old male), image B) represents the 'middle-aged' group (44-year-old male), and image C) represent the 'old' group (69-year-old male). $\mathrm{M}=$ the location of medial facet; $\mathrm{N}=$ the intercondylar notch; $\mathrm{L}=$ the lateral facet

Figure 2 The Bland-Altman plots demonstrate the mean difference between the cartilage thickness measurements at visit 2 and visit 1 (i.e. visit 2 minus visit 1) plotted against the mean of the two visits (i.e. visit 1 plus visit 2, divided by 2). Plot A) represents intercondylar notch, B), lateral facet and C) medial facet (solid line represents mean difference and dashed lines represent upper and lower limits of agreement)

Figure 3 Variation of mean femoral cartilage thickness at the intercondylar notch, lateral facet and medial facet with physical characteristics of the participants. A) Age, B) Height, C) Weight, and D) BMI. R-value, significance value, and regression equation are also presented above with significant findings highlighted in bold. Solid, dashed and round dot trendline $=$ intercondylar notch, lateral facet, and medial facet, respectively

Figure 4 Presents variation of mean femoral cartilage thickness at the intercondylar notch, lateral facet and medial facet with physical characteristics of the participants for both males and females A) age, B) height, C)

Weight, and D) BMI. R-value, significance value, and regression equation are also presented above with

500 significance highlighted in bold. Black trendline = male; grey trendline $=$ female; Solid line $=$ notch; dashed trendline $=$ lateral; round dot trendline $=$ medial

503 Figure 5 Mean cartilage thickness measurements at the medial facet, intercondylar notch and the lateral facet: * $=$ significant difference between groups at $\mathrm{P}<0.01$ level. Data are means $\pm \mathrm{SD}$

506 Figure 6 Mean cartilage thickness measurements at the medial facet, intercondylar notch and the lateral facet

507 for both male $(\mathrm{n}=17)$ and female $(\mathrm{n}=17)$ participants, matched for age and BMI. * = significant difference

508 between groups at $\mathrm{P}<0.01$ level. Data are means $\pm \mathrm{SD}$ 

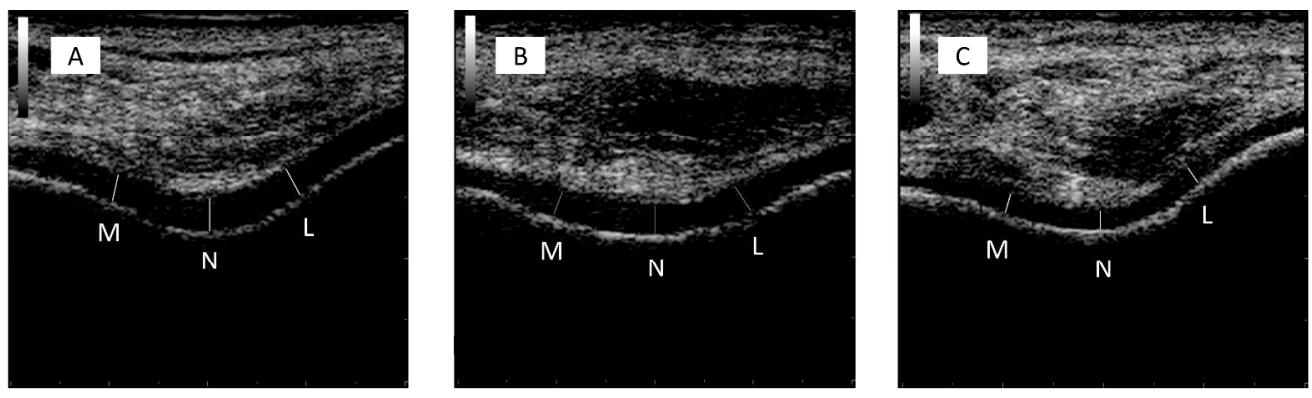

Figure 1. US transverse image of the femoral articular cartilage demonstrating the difference in image quality and clarity between young, middle-aged, and old groups. Image A) represents the 'young' group (23-year-old male), image B) represents the 'middle-aged' group (44-year-old male), and image C) represent the 'old' group (69-year-old male). $\mathrm{M}=$ the location of medial facet; $\mathrm{N}=$ the intercondylar notch; $\mathrm{L}=$ the lateral facet 
A

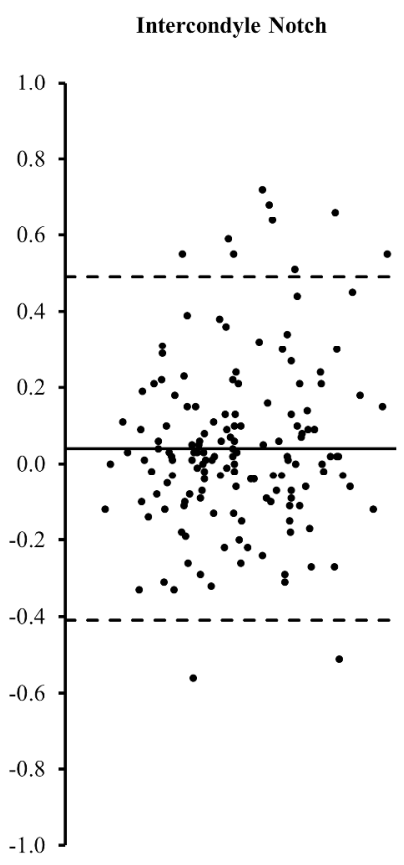

B

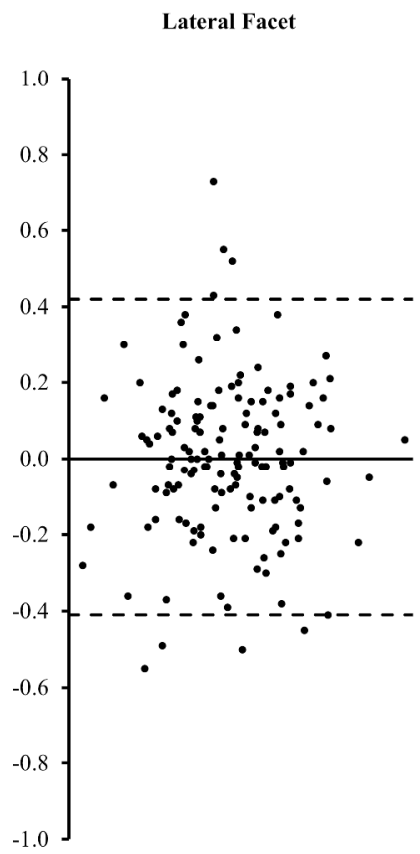

C

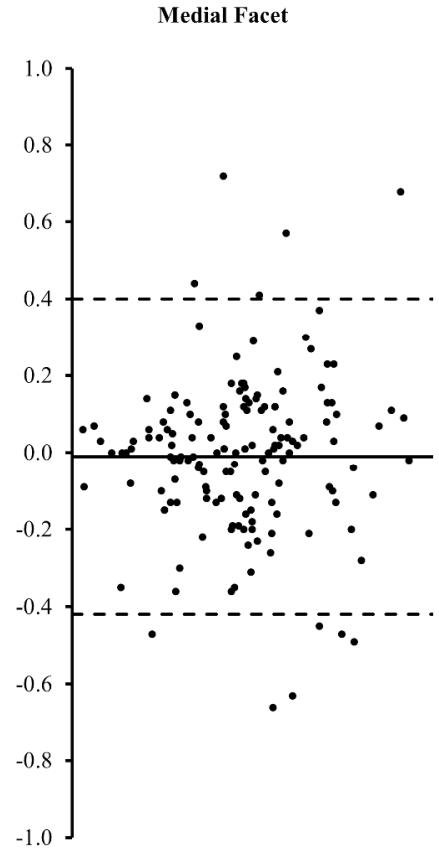

Figure 2. The Bland-Altman plots demonstrate the mean difference between the cartilage thickness measurements at visit 2 and visit 1 (i.e. visit 2 minus visit 1) plotted against the mean of the two visits (i.e. visit 1 plus visit 2, divided by 2 ). Plot A) represents intercondylar notch, B), lateral facet and C) medial facet (solid line represents mean difference and dashed lines represent upper and lower limits of agreement) 

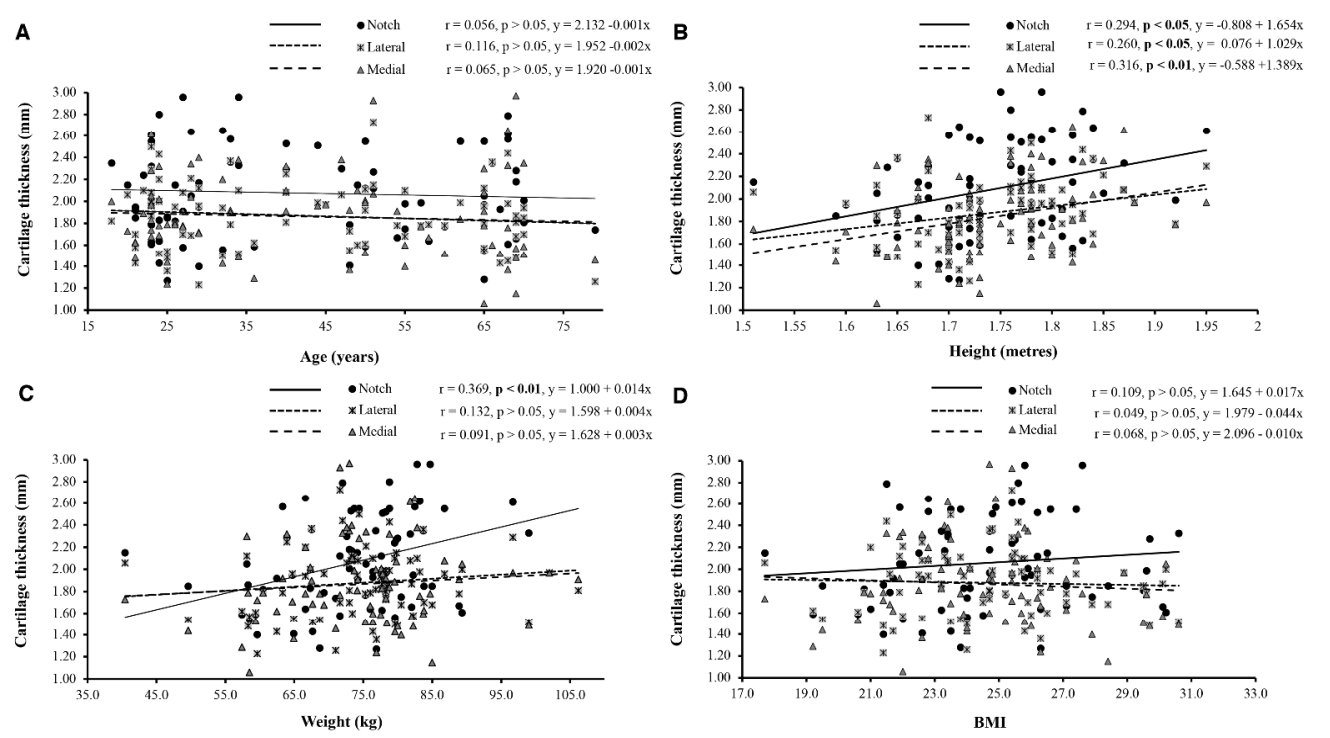

Figure 3. Variation of mean femoral cartilage thickness at the intercondylar notch, lateral facet and medial facet with physical characteristics of the participants. A) Age, B) Height, C) Weight, and D) BMI. R-value, significance value, and regression equation are also presented above with significant findings highlighted in bold. Solid, dashed and round dot trendline = intercondylar notch, lateral facet, and medial facet, respectively 

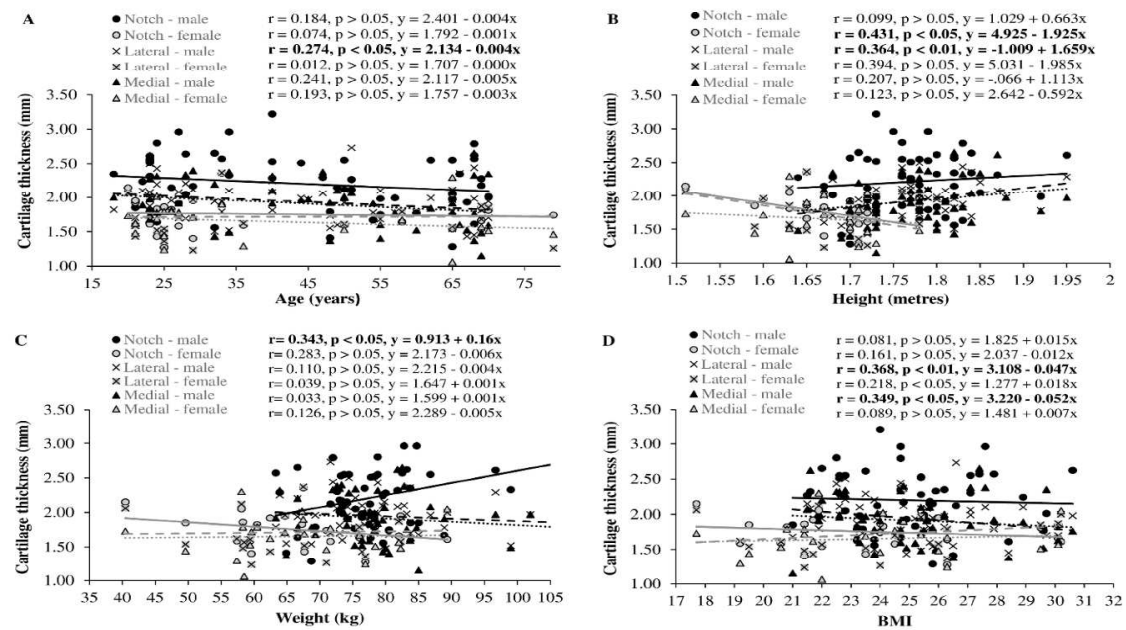

Figure 4. Presents variation of mean femoral cartilage thickness at the intercondylar notch, lateral facet and medial facet with physical characteristics of the participants for both males and females A) age, B) height, C) Weight, and D) BMI. R-value, significance value, and regression equation are also presented above with significance highlighted in bold. Black trendline = male; grey trendline = female; Solid line = notch; dashed trendline $=$ lateral; round dot trendline $=$ medial 


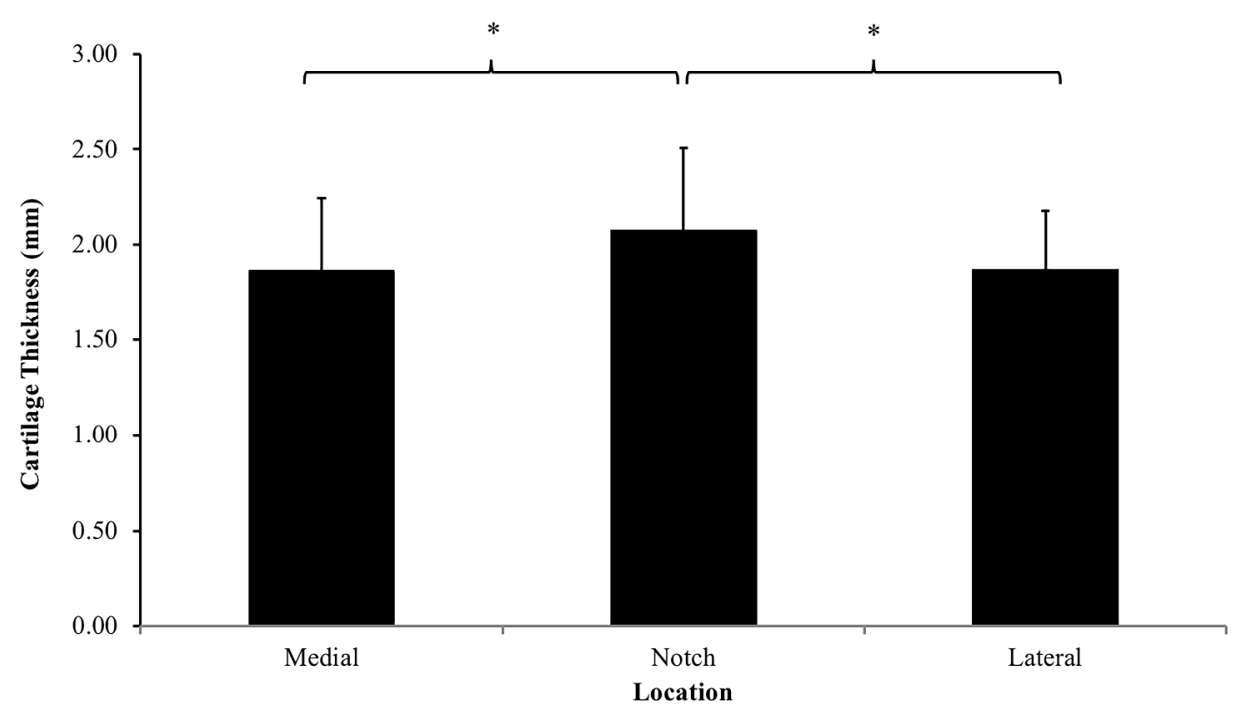

Figure 5. Mean cartilage thickness measurements at the medial facet, intercondylar notch and the lateral facet: $*=$ significant difference between groups at $\mathrm{P}<0.01$ level. Data are means \pm SD 


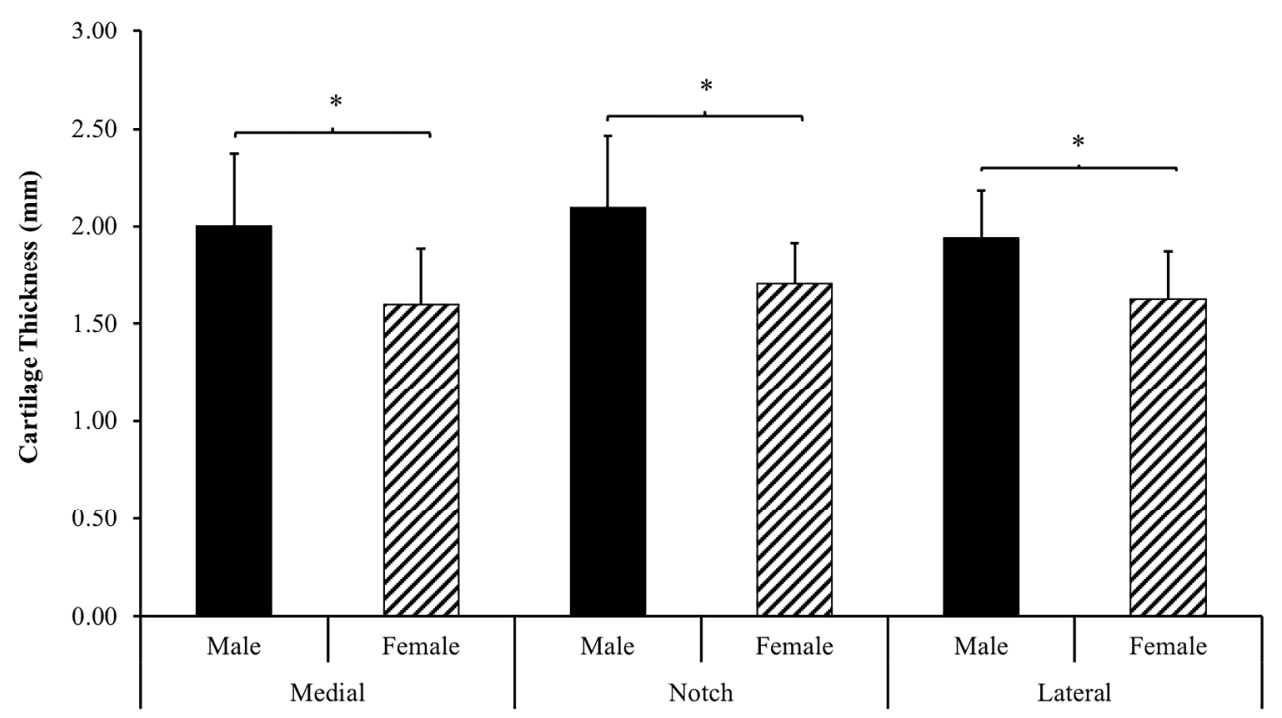

Figure 6. Mean cartilage thickness measurements at the medial facet, intercondylar notch and the lateral facet for both male $(n=17)$ and female $(n=17)$ participants, matched for age and BMI. * = significant difference between groups at $P<0.01$ level. Data are means \pm SD 